\title{
Inadequate Diagnosis and Treatment of Malaria Among Travelers Returning from Africa During the Ebola Epidemic — United States, 2014-2015
}

\author{
Kathrine R. Tan, $\mathrm{MD}^{1}$; Karen A. Cullen, $\mathrm{PhD}^{1}$; Emilia H. Koumans, $\mathrm{MD}^{2}$; Paul M. Arguin, $\mathrm{MD}^{1}$
}

Among 1,683 persons in the United States who developed malaria following international travel during 2012, more than half acquired disease in one of 16 countries* in West Africa (1). Since March 2014, West Africa has experienced the world's largest epidemic of Ebola virus disease (Ebola), primarily affecting Guinea, Sierra Leone, and Liberia; in 2014, approximately 20,000 Ebola cases were reported (2). Both Ebola and malaria are often characterized by fever and malaise and can be clinically indistinguishable, especially early in the course of disease. Immediate laboratory testing is critical for diagnosis of both Ebola and malaria, so that appropriate lifesaving treatment can be initiated. CDC recommends prompt malaria testing of patients with fever and history of travel to an area that is endemic for malaria, using blood smear microscopy, with results available within a few hours (3). Empiric treatment of malaria is not recommended by CDC (4). Reverse transcription-polymerase chain reaction (RT-PCR) testing is recommended to diagnose Ebola (5). During the Ebola outbreak in West Africa, CDC received reports of delayed laboratory testing for malaria in travelers returning to the United States because of infection control concerns related to Ebola (๑). CDC reviewed documented calls to its malaria consultation service and selected three patient cases to present as examples of deficiencies in the evaluation and treatment of malaria among travelers returning from Africa during the Ebola epidemic.

Malaria parasites can be detected by microscopic examination of a Giemsa-stained drop of the patient's blood (a blood smear). CDC recommends that both thick and thin blood smears be obtained immediately for all febrile patients who have a compatible travel history, regardless of other associated symptoms, and that results be available within hours (3). Malaria can be conclusively ruled out in 24 hours by three negative smears collected at 12-hour intervals. Blood smears also provide information about the infecting species and level of parasitemia (percentage of infected red blood cells), which, along with signs and symptoms, determine appropriate antimalarial treatment. Severe malaria, defined as the presence of $\geq 5 \%$ of infected red blood cells, or at least one of several complications, ${ }^{\dagger}$ should be treated with

\footnotetext{
* Benin, Burkina Faso, Cape Verde, Côte d'Ivoire, Gambia, Ghana, Guinea, GuineaBissau, Liberia, Mali, Mauritania, Niger, Nigeria, Senegal, Sierra Leone, and Togo. $\dagger$ Severe malaria is defined as the presence of at least one of the following in a patient with malaria: acidosis, acute respiratory distress syndrome, seizures, disseminated intravascular congestion, hyperparasitemia ( $\geq 5 \%$ parasitemia), hypoglycemia, impaired consciousness, jaundice, acute renal injury, severe anemia (hemoglobin $<7 \mathrm{~g} / \mathrm{dL}$ ), or shock.
}

intravenous antimalarials (4). Treatment of malaria without information from the blood smear can lead to poor outcomes because of incorrect antimalarial selection, inappropriate treatment of severe malaria with oral antimalarials, and misdiagnosis of other febrile illnesses that are not malaria. Empiric treatment of malaria is not recommended (4).

Since October 2014, travelers from countries with ongoing Ebola virus transmission have been screened upon arrival at U.S. airports to ascertain risk factors and signs and symptoms of Ebola, and are assigned to one of four risk categories. ${ }^{\S}$ Healthy travelers who are classified as having "low but not zero" risk for Ebola are actively monitored by state or local public health authorities; travelers must check their temperature twice daily for 21 days after arrival, and must call the health department for evaluation if symptoms or temperature $\geq 100.4^{\circ} \mathrm{F}$ occur.9 A person with fever or symptoms suggestive of Ebola who had an epidemiologic risk factor within 21 days before symptom onset is considered a person under investigation for Ebola. ${ }^{* *}$ Because malaria is endemic year-round and countrywide in the countries where Ebola transmission is occurring (3), persons who have fever and are under investigation for Ebola should always receive immediate malaria testing (7).

At CDC, the Malaria Branch of the Division of Parasitic Diseases and Malaria conducts malaria surveillance and provides clinical consultation for the diagnosis and management of malaria. Through this consultation service, CDC became aware of delays in malaria diagnosis and treatment related to concerns about Ebola. Three case reports are presented to illustrate inadequate diagnosis and treatment of malaria in persons who traveled to Africa during the Ebola epidemic.

\section{Case 1}

In March 2015, a man aged 34 years entered the United States after visiting Sierra Leone, Guinea, and Senegal. He was afebrile, classified as having low but not zero risk for Ebola, and was enrolled in the active monitoring process. Seven days after returning to the United States (day 1 of illness), he developed nausea, anorexia, and a $105.8^{\circ} \mathrm{F}$ fever; early that morning, he

\footnotetext{
$\$$ http://www.cdc.gov/vhf/ebola/exposure/risk-factors-when-evaluating-personfor-exposure.html.

Ittp://www.cdc.gov/vhf/ebola/exposure/monitoring-and-movement-ofpersons-with-exposure.html.

** http://www.cdc.gov/vhf/ebola/healthcare-us/evaluating-patients/casedefinition.html.
} 
called emergency medical services (EMS) rather than the local health department. When he told EMS responders his travel history while en route to the hospital, the responders stopped the vehicle, donned personal protective equipment, and then proceeded to hospital A, where the patient was placed in isolation and was given oral medications for his fever. Blood was drawn for malaria testing, but the laboratory would not process the specimens, citing concern about possible Ebola exposure. The state laboratory agreed to test the specimens.

At 3:00 p.m. that afternoon, malaria PCR test results were determined to be positive, and Ebola RT-PCR results and influenza test results of a nasopharyngeal swab were both negative. The state health department and CDC advised hospital A to begin antimalarial treatment immediately. Because blood smear microscopy was not done, it was not known whether the patient had hyperparasitemia ( $\geq 5 \%$ parasitemia), which is one sign of severe malaria, and for which parenteral antimalarials are indicated. However, because hospital staff members feared Ebola, they were not comfortable placing an intravenous catheter. The patient was given an oral antimalarial (artemetherlumefantrine) on the evening of day 1 , and was transferred to hospital B on day 2, where a thin smear confirmed Plasmodium falciparum malaria with a $2.5 \%$ parasitemia. He completed oral therapy, had no complications, and was discharged 3 days later.

\section{Case 2}

In March 2015, 1 day before traveling to the United States from Kenya, a man aged 69 years developed subjective fever. No Ebola transmission has been reported in Kenya. On the third day of fever, he visited an urgent-care clinic and reported his recent travel to Kenya. No tests were performed, and the patient was given a prescription for the antimalarial mefloquine for empiric treatment of suspected malaria. He was unable to fill the prescription because local pharmacies did not have the medication in stock.

The man continued to have fever, myalgias, and weakness, and went to an emergency department (ED) at midday. Blood was drawn for malaria testing, but malaria microscopy services were not available on weekends. The patient was released from the ED and told that the laboratory results would be available in 2 days. He received no treatment. His fever persisted, and his weakness increased; at midnight he visited a different ED, again reporting his travel to Kenya. Because of his history of travel to Africa, he was placed in isolation and his medical assessment was suspended for the next 4 hours until the hospital staff members were assured that a traveler from Kenya was not at risk for Ebola. Blood smear microscopy was positive for $P$. falciparum, but the level of parasitemia was not reported. He was treated with oral atovaquone-proguanil and discharged later that morning. He completed his antimalarial treatment and recovered with no complications.

\section{Case 3}

In May 2015, a woman aged 31 years returned to the United States from Sierra Leone and visited an ED with fever and abdominal pain. The hospital laboratory refused to perform any diagnostic testing, including malaria smears, until a diagnosis of Ebola was ruled out. After discussions involving clinicians, the state health department, and CDC, and hours after arrival, a malaria rapid diagnostic test that had been approved by the Food and Drug Administration for laboratory use only was performed at bedside and was negative. Following a negative Ebola RT-PCR result 9 hours later, other laboratory tests were performed, leading to the diagnosis of a urinary tract infection. A malaria smear was not performed. The clinical outcome for this patient is not known.

\section{Discussion}

Malaria is a common cause of fever among travelers who have been to areas where the disease is endemic. Patients in whom a diagnosis of malaria is suspected should be urgently evaluated. One study evaluating the etiology of fever among returned travelers seeking care at a multicenter, multinational travel clinic network found malaria to be the most common single etiologic diagnosis, accounting for $21 \%$ of all diagnoses (8). Health care providers should ask patients with fever about places of recent travel. Febrile persons with history of travel to a malaria-endemic area should be tested for malaria with blood smear microscopy without delay, irrespective of whether travel occurred in an Ebola-affected country. Although current recommendations for preparing malaria smears remain the standard (9), CDC has developed a Giemsa staining procedure that inactivates viruses, including Ebola virus, during slide preparation to increase the safety of this testing procedure ( 7 ).

These three case reports illustrate inappropriate practices in evaluation and management of febrile travelers and inadequate diagnosis and treatment for malaria because of concerns about possible exposure to Ebola. In case 1, the hospital laboratory's reluctance to process the patient's blood specimen introduced delay in malaria testing, and PCR testing rather than blood smear testing for malaria was performed at the state laboratory. Furthermore, intravenous access is of paramount importance to deliver fluids and medications in dehydrated or very ill patients; therefore, unrestricted access to parenteral interventions was important should the patient's condition have deteriorated. In case 2, a prescription for empiric malaria treatment was provided without laboratory diagnosis, contrary to CDC recommendations (4). Furthermore, delayed malaria testing occurred when clinical assessment was halted until hospital staff 


\section{Summary}

What is already known on this topic?

Malaria cases are rarely diagnosed in the United States; however, malaria is potentially fatal if the diagnosis or treatment, or both, are delayed. Febrile travelers who recently visited a malaria-endemic area should be tested for malaria without delay by blood smear microscopy, with results available within hours. Empiric treatment of malaria is not recommended.

What is added by this report?

During the Ebola epidemic, there were deficiencies in malaria diagnosis, treatment, and laboratory practices in the United States related to concerns about exposure of laboratory and clinical staff members to Ebola.

What are the implications for public health practice?

Malaria evaluation should be prioritized in febrile persons who travelled to malaria-endemic areas regardless of travel to an Ebola-affected country. Timely and immediate education is needed for health care providers and laboratory managers to encourage adherence to guidelines for evaluation and management of malaria in the febrile traveler to prevent poor outcomes.

members could be reassured that the patient was not at risk for Ebola. In case 3, Ebola infection control fears led to the use of a bedside rapid diagnostic test to rule out malaria, despite this test not being approved by the Food and Drug Administration for point-of-care testing. Using a rapid diagnostic test to rule out malaria instead of blood smear microscopy is also contrary to CDC recommendations (4).

An internal review of Ebola-related inquiries to CDC found that 1) recommended steps in the evaluation of febrile persons who traveled to an area with endemic malaria (3) were followed in the evaluation of fewer than one third of febrile travelers, regardless of whether they had come from an Ebola-affected country; 2) although intravenous antimalarials are recommended for all patients with severe malaria to rapidly reduce parasitemia, increase the probability of survival, and decrease the likelihood of complications (10), only one third of patients with severe malaria received intravenous antimalarials; and 3) more than one third of the antimalarials received by travelers were prescribed empirically (Division of Parasitic Diseases and Malaria, Center for Global Health, CDC, unpublished data, 2014-2015).

The findings in this report are subject to at least two limitations. First, the case reports were selected intentionally to illustrate the occurrence of suboptimal practices; however, the prevalence of these practices is not known. Second, the cases described were reconstructed from consultation notes, and clinical details were missing for some of these cases, such as the outcome of case 3 .

These selected case reports indicate inadequate implementation of current malaria diagnostic and treatment guidelines among febrile travelers who had been to malaria-endemic countries, related, in part, to health care provider and laboratory concerns about risks for possible exposure to Ebola during diagnostic evaluations and clinical procedures. It is important that all febrile patients with history of travel to a malaria-endemic country be tested for malaria as soon as possible using blood smear microscopy, regardless of their other risk factors, with results available within hours (3). Further information on safe diagnosis of malaria can be found in CDC's Guidance for Malaria Diagnosis in Patients Suspected of Ebola Infection in the United States (7). It is also critical that after receipt of a positive malaria test result, appropriate antimalarials, including parenteral antimalarials for severe malaria, be started without delay (4). Empiric treatment of malaria is not recommended (4).

\section{Acknowledgments}

Shirley Lecher, Achala Jayatelleki, Eyal Leshem, Christopher Lehmann, Elliot Raizes, Paul Mead, CDC.

\footnotetext{
${ }^{1}$ Division of Parasitic Diseases and Malaria, Center for Global Health, CDC; ${ }^{2}$ Divison of Reproductive Health, National Center for Chronic Disease Prevention and Health Promotion, CDC.

Corresponding author: Kathrine R. Tan, ktan@cdc.gov, 404-718-4701.
}

\section{References}

1. Cullen KA, Arguin PM. Malaria surveillance-United States, 2012. MMWR Surveill Summ 2014;63:1-22.

2. CDC. 2014 Ebola outbreak in West Africa: case counts. Atlanta, GA: US Department of Health and Human Services, CDC; 2014. http:// www.cdc.gov/vhf/ebola/outbreaks/2014-west-africa/previous-casecounts.html.

3. CDC. Health information for international travel 2016. New York, NY: Oxford University Press; 2016.

4. CDC. Treatment of malaria (guidelines for clinicians). Atlanta, GA: US Department of Health and Human Services, CDC; 2013. http://www. cdc.gov/malaria/resources/pdf/clinicalguidance.pdf.

5. CDC. Guidance for collection, transport and submission of specimens for Ebola virus testing. Atlanta, GA: US Department of Health and Human Services, CDC; 2015. http://www.cdc.gov/vhf/ebola/healthcareus/laboratories/specimens.html.

6. Karwowski MP, Meites E, Fullerton KE, et al. Clinical inquiries regarding Ebola virus disease received by CDC_-United States, July 9-November 15, 2014. MMWR Morb Mortal Wkly Rep 2014;63:1175-9.

7. CDC. Guidance for malaria diagnosis in patients suspected of Ebola infection in the United States. Atlanta, GA: US Department of Health and Human Services, CDC; 2015. http://www.cdc.gov/malaria/new_ info/2014/malaria_ebola.htm.

8. Wilson ME, Weld LH, Boggild A, et al. Fever in returned travelers: results from the GeoSentinel Surveillance Network. Clin Infect Dis 2007;44:1560-8. http://dx.doi.org/10.1086/518173.

9. CDC. Laboratory diagnosis of malaria: staining for malaria parasites. Atlanta, GA: US Department of Health and Human Services, CDC; 2015. http://www.cdc.gov/dpdx/resources/pdf/benchAids/malaria/ malaria_staining_benchaid.pdf.

10. Griffith KS, Lewis LS, Mali S, Parise ME. Treatment of malaria in the United States. JAMA 2007;297:2264-77. http://dx.doi.org/10.1001/ jama.297.20.2264. 